\title{
Identification and Functional Characterization of Bicaudal-D2 As A \\ Candidate Disease Gene in Autosomal Recessive Consanguineous
}

\section{Family with Dilated Cardiomyopathy}

Kai Luo $\ddagger^{1,2}$, Chenqing Zheng $\ddagger^{3}$, Rong $\mathrm{Luo}^{4}$, Xin $\mathrm{Cao}^{5}$, Huajun Sun ${ }^{2,6}$, Huihui $\mathrm{Ma}^{1,2}$, Jichang Huang ${ }^{4}, \mathrm{Xu}$ Yang ${ }^{3}$, Xiushan $\mathrm{Wu}^{7^{*}}$, Xiaoping $\mathrm{Li}^{1,2^{*}}$

$\ddagger$ These authors contributed equally to this work.

1 Department of Cardiology, Sichuan Provincial People's Hospital, University of Electronic Science and Technology of China, Chengdu, Sichuan 610072, China

2 Chinese Academy of Sciences Sichuan Translational Medicine Research Hospital, Chengdu 610072, China

3 Shenzhen Aone Medical Laboratory Co., Ltd., Shenzhen, China

4 Institute of Geriatric Cardiovascular Disease, Chengdu Medical College, Chengdu, People's Republic of China

5 School of Acupuncture-Moxibustion and Tuina, Chengdu University of Traditional Chinese Medicine, Chengdu, China

6 Department of Pathology, Sichuan Provincial People's Hospital, University of Electronic Science and Technology of China, Chengdu, Sichuan 610072, China

7 The Center for Heart Development, Hunan Normal University, Changsha, China

*, Authors for correspondence. Email: Xiushan Wu, xiushanwu2003@aliyun.com;

Xiaoping Li, lixiaoping0119@163.com. 


\section{Abstract}

Familial Dilated cardiomyopathy (DCM) is a genetic cardiomyopathy with reduced left ventricle function or systolic function. Fifty-one DCM genes have been reported, most of which are inherited in an autosomal dominant manner, while those caused by a recessive manner are rarely observed. Here we identified an autosome recessive and evolutionarily conserved missense variant, c.2429G>A: p.Arg810His, in bicaudal D homolog 2 (BICD2), which was segregated with the disease phenotype in a consanguineous family with DCM. Furthermore, we confirmed the presence of BICD2 variants in 3 of 210 sporadic cases, highlighting its candidate causative role. Interestingly, we discovered that $B I C D 2$ expressed higher in cardiomyocytes from DCM than that from control by reanalyzing published scRNA-seq dataset, implicating its possible involvement in cardiac function. We next explored BICD2 function in zebrafish model at both embryonic and adult stages. Knockout of bicd2 resulted in partial embryonic lethality in homozygotes, suggesting a vital role for bicd 2 in embryogenesis. We performed zebrafish echocardiography to detect indices of ventricular size at the adult zebrafish stage. Intriguingly, dilated hearts, decreased ejection fraction, cardiac output and stroke volume were observed, suggesting a phenotype similar to human DCM in bicd2-knockout homozygotes. Furthermore, RNA-seq confirmed the largest transcriptome shift in bicd2 homozygotes. Gene Set Enrichment Analysis(GSEA) of bicd2-deficient fish showed altered gene expression enriched in cardiac pathways and mitochondrial energy metabolism. In conclusion, we found for the first time that an autosomal recessive BICD2 variant is associated with familial DCM, providing further insight into the molecular pathological mechanism of DCM.

Keywords: dilated cardiomyopathy, BICD2, zebrafish model, RNA-seq 


\section{Introduction}

Cardiomyopathy is a heterozygous disease of the heart muscle, leading to heart failure. Cardiomyopathy mainly include dilated cardiomyopathy(DCM), hypertrophic cardiomyopathy(HCM), as well as arrhythmogenic right ventricular cardiomyopathy (ARVC)[1]. DCM is a cardiomyopathy characterized by dilatation of the left ventricle or bilateral ventricles with myocardial systolic dysfunction[2]. The prevalence of dilated cardiomyopathy reported in global is $1 / 250$ [3]. It is the third leading cause of heart failure and the most common cause of heart transplantation [4], but its etiology and pathogenesis remains elusive.

Familial DCM is now more commonly diagnosed, owing to the improved awareness of disease-causing genetic variants and genetic screening. Familial DCM is found in $20-35 \%$ of patients with DCM, and $80 \%$ of DCM patients inherited in autosomal dominant pattern, $10 \%-15 \%$ of DCM patients belong to autosomal repressive or X-linked inheritance[5]. Previous DCM studies unraveled >250 associated genes spanning $>10$ gene ontologies, suggesting a complex and diverse genetic architecture[6]. A DCM Gene Curation Expert Panel curated a final set of 51 genes proposed to have a monogenic role in isolated, idiopathic DCM in humans[6]. And 12 out of 51 genes, ie. BAG3, DES, FLNC, LMNA, MYH7, PLN, RBM20, SCN5A, TNNC1, TNNT2, TTN, DSP were classified as having definitive or strong evidence[6]. Other 7 genes including ACTC1, ACTN2, JPH2, NEXN, TNNI3, TPM1, and VCL were classified as moderate evidence[6]. Although assessing as having high evidence, these 19 genes explain only a minority of cases, leaving the remainder of DCM genetic architecture incompletely addressed[6].

Most reported DCM variants inherited in an autosomal dominant pattern[5-7], while autosomal recessive(AR) inheritance in familial DCM was less frequently observed and implicated in pediatric DCM[8-10]. Consanguineous families is ideal study objects for investigating AR DCM. However, few researches focused on familial 
DCM in consanguineous families[10-12].

Bicaudal-D2 (BICD2) is a dynein activating adaptor protein that plays a critical role in microtubule-based minus-end-directed transport[13, 14]. Mutations in the human BICD2 have been linked to a spectrum of neuronal disorders, in particular to a dominant mild early onset form of spinal muscular atrophy[15-18]. The BICD2 protein harbors a C-terminus recognizing and binding to the material being transported and an N-terminus containing three helix-helix structural domains that bind to the kinesin tail and kinesin-activating protein filaments to form a sandwich structure of the kinesin complex, which activates the kinesin and allows the kinesin complex to travel along the microtubule cytoskeleton to transport cargo[19]. Bicd2-KO mice showed significantly lower LMNA expression at protein level, decreased $M K L-1 / S R F$ activity, and significantly downregulated expression of its downstream $\alpha$-actin, $\alpha$-coactin and membrane association proteins, and microtubulin[20]. $M K L-1$, a member of the actin family, plays an important role in cardiovascular system development and function as a cofactor of serum response factor $(S R F)$ that activates $S R F$-dependent transcriptional regulatory elements. In nuclear fibrillar protein A/C gene (LMNA) knockout mice, the MKL1/SRF signaling pathway is also inhibited, and endogenous $M K L-1$ nuclear translocation in cardiomyocytes is dysfunctional, with reduced expression of $S R F, \alpha$-actinin and membrane association proteins, leading to the development of dilated heart disease[21] . Simple blockade of $S R F$ expression in mouse myocardial tissue can lead to the development of DCM[22].

Zebrafish model is one of the ideal models to study the function of mutated genes in DCM, and harbored an enlarged heart, reduced shortening fraction during cardiac contraction and no cardiomyocyte proliferation, consistent with the characteristics of human DCM[23].

In this study, we performed whole-exome sequencing (WES) in consanguineous 
family DCM to unravel DCM-causing gene. Subsequently, functional studies of candidate DCM gene, $B I C D 2$, were conducted in zebrafish models to demonstrate the relationship between BICD2 and DCM.

\section{Materials and Methods}

\section{Patients and clinical evaluation}

The proband and zir families from consanguineous family was recruited in 2011. In total 210 sporadic DCM patients who hospitalized at the Cardiology Department of Sichuan People's Hospital from 2014 to 2017, were enrolled for further BICD2 variant validation. This study was approved by the Institutional Research Committee of Sichuan Provincial People's Hospital. Written informed consent was obtained from all subjects participating.

\section{Genomic DNA preparation}

Peripheral blood from DCM cases was collected into EDTA anticoagulant tubes, and genomic DNA was extracted using a blood DNA extraction kit according to the manufacturer's protocol (Qiagen, Germantown, MD, USA).

\section{Exome sequencing and bioinformatics analysis}

To identify additional genes for DCM, we applied WES to search for potential genetic variants in DCM family without pathologic variants in known DCM genes. WES was performed on four members of family 1(the proband, sibling of the proband, parents of the proband). The normal human population database consisted of The Thousand Genomes Project (http://browser.1000genomes. org), ESP6500SI-V2 (http://evs.gs.was

-hington.edu/EVS) , ExAC Human Exome Integration Database (http://exac.broadinstit -ute.org/) and the sequencing company's internal database. All variants were annotated with ANNOVAR software (version 2014). 


\section{Quantitative RT-PCR}

Total RNA was isolated from cells using the Qiagen RNeasy Mini kit. In parallel, we analyzed the mRNA concentration of the housekeeping $\beta$-actin as an internal control for normalization. The real-time monitoring of the PCR reaction, the precise quantification of the products in the exponential phase of the amplification and the melting curve analysis were performed with the Bio-Rad CFX Manager software, following recommended instructions of the manufacturer.

\section{Immunocytochemistry}

Heart tissue fixed with $4 \%$ paraformaldehyde was dehydrated by an automatic dehydrator, embedded, and sectioned as the following protocols. Firstly, the dewaxed sections were placed in a staining vat with $3 \%$ methanolic hydrogen peroxide at room temperature for $10 \mathrm{~min}$. Secondly, PBS wash 3 times (5 min each time). Then, immersing the sections in $0.01 \mathrm{M}$ citrate buffer ( $\mathrm{PH} 6.0$ ), heating to boiling using Microwave Oven and then disconnecting, after an interval of 5 min, repeated once, after cooling, washed 2 times with PBS (5 min each time). Adding normal goat serum blocking solution dropwise, room temperature for 20 min. Adding primary antibody dropwise, overnight at $4^{\circ} \mathrm{C}$. Adding biotinylated secondary antibody dropwise, $37^{\circ} \mathrm{C}$ for 30 min. PBS wash 3 times (5 min each time). Mixing the reagents of DAB Color Development Kit (K135925C, Beijing Zhongshan Jinqiao Biotechnology Co., LTD), and add dropwise to the sections at room temperature, for about $2 \mathrm{~min}$, then wash with distilled water. Hematoxylin lightly re-stained, dehydrated, transparent, and sealed with neutral gum. Epifluorescence Images were acquired using an BA200 Trinocular Microscope (McAudi Industrial Group Co., Ltd).

\section{CRISPR/Cas9 bicd2 knock out in zebrafish}

Considering the conserved functional domain of the protein encoding by bicd2, we designed CRISPR sequence to target the genomic sequence corresponding after coding 80 amino acids, so as to disrupt the conserved structural domain of the BicD 
medRxiv preprint doi: https://doi.org/10.1101/2022.02.03.22269864; this version posted February 4, 2022. The copyright holder for this preprint (which was not certified by peer review) is the author/funder, who has granted medRxiv a license to display the preprint in perpetuity.

All rights reserved. No reuse allowed without permission.

superfamily. And it is recommended that the CRISPR sequence be designed in Exon 2. Four guide RNA targeting the bicd2 locus was designed following criteria of high on-target score and low off-target score. Zebrafish fertilized eggs were collected. Cas9 protein(a final concentration of $200 \mathrm{ng} / \mathrm{ul}$ ) were mixed with sgRNAs (a final concentration of $\sim 80 \mathrm{ng} / \mathrm{ul}$ ) and subsequently microinjected into zebrafish fertilized eggs ( $1 \mathrm{nl}$ per embryo). Five embryos were taken when the injected embryos developed to $24 \mathrm{hpf}$, and genomic DNA templates were prepared under the following reaction conditions: $65^{\circ} \mathrm{C}$ for $30 \mathrm{~min}, 95^{\circ} \mathrm{C}$ for $5 \mathrm{~min}, 16^{\circ} \mathrm{C}$ for $1 \mathrm{~min}, 4^{\circ} \mathrm{C}$ for $2 \mathrm{~min}$. PCR amplification products were cloned into pGEM-T Easy plasmid (5 $\mu$ l total volume of reaction). The above $5 \mu \mathrm{l}$ ligation product was transformed into $50 \mu \mathrm{E}$. coli DH5 $\alpha$ receptor cells (pfu $\geq 108$ ). Then coated in LB plates containing ampicillin $(50 \mu \mathrm{g} / \mathrm{ml})$ and incubated upside down overnight at $37^{\circ} \mathrm{C}$. Positive clones were validated by Sanger sequencing. Experimental results confirmed that sgRNA1 and sgRNA2 could effectively guide Cas9 to target cleavage of bicd2.

bicd2-sgRNA1 :

GGUUGAGUGAACCUGGCCAUGUUUUAGAGCUAGAAAUAGCAAGUUAAA AUAAGGCUAGUCCGUUAUCAACUUGAAAAAGUGGCACCGAGUCGGUGC UUUUUUU

bicd2-sgRNA2 :

GGAGUCGCUCAUCCUGGAGUGUUUUAGAGCUAGAAAUAGCAAGUUAAA AUAAGGCUAGUCCGUUAUCAACUUGAAAAAGUGGCACCGAGUCGGUGC UUUUUUU

bicd2-sgRNA3 :

GUACUAUGAGCAGAGGGUGCGUUUUAGAGCUAGAAAUAGCAAGUUAAA AUAAGGCUAGUCCGUUAUCAACUUGAAAAAGUGGCACCGAGUCGGUGC UUUUUUU

bicd2-sgRNA4 : 
GCUCGAGGGCAAGGGUGGCCGUUUUAGAGCUAGAAAUAGCAAGUUAAA AUAAGGCUAGUCCGUUAUCAACUUGAAAAAGUGGCACCGAGUCGGUGC UUUUUUU

\section{Zebrafish echocardiogram}

The Vevo2100® Imaging System and Vevo Imaging Station (VisualSonics) was used to perform transthoracic echocardiography on 7-month-old zebrafish to examine indicators of ventricular function and ventricular size during systole and diastole with a 22-55 MHz (MS700) transducer probe. Before echocardiography, zebrafish were anesthetized with $0.02 \%$ tricaine (MS-222) to induce sedation without cessation of breathing. Anesthetized fish were then transferred into a glass beaker containing a weighted sponge with a groove. The fish was fixed on a sponge in the supine position. Echocardiography were collected and analyzed from the apical 2-chamber view. We typically completed image acquisition within 5 min of inducing anesthesia. After echocardiography, fish were placed in an aerated recovery chamber containing fresh system water without anesthetic and they recovered within $30 \mathrm{~s}$ to $2 \mathrm{~min}$, and there were no deaths. The two-dimensional B-mode was used for measurement of heart rate, ejection fraction, fractional shortening, end-diastolic and end-systolic area, end-diastolic and end-systolic volume, cardiac output, and stoke volume. The left ventricular volumes are calculated by tracing the endocardial border manually at end diastole and at end systole (planimetry). B-mode imaging quality was further optimized by adjusting focal depth, gain, image width and depth.

\section{RNA-seq and data analysis}

Total RNA from zebrafish hearts was isolated from cells using the Qiagen RNeasy Mini kit. Qubit2.0 Fluorometer was used to measure the quality and concentration of mRNA. Commercial sequencing companies sequenced our mRNA samples by Illumina Hiseq $\mathrm{X}$ and returned raw sequencing reads to us. Before further data analysis, we firstly checked raw data quality and removed reads with poor quality to get clean reads with high quality. Then all clean reads were mapped to zebrafish 
medRxiv preprint doi: https://doi.org/10.1101/2022.02.03.22269864; this version posted February 4, 2022. The copyright holder for this preprint (which was not certified by peer review) is the author/funder, who has granted medRxiv a license to display the preprint in perpetuity.

All rights reserved. No reuse allowed without permission.

genome Assembly GRCz11 (https://hgdownload.soe.ucsc.edu/goldenPath/danRer11/ bigZips/genes/danRer11.ensGene.gtf.gz) using HISAT (version 2.2.1) [24]. StringTie (version 2.2.0)[24] was used to quantify gene expression in different samples and FPKM values were extracted as the expression metric. EBSeq (version 1.34.0 ) [25] was utilized on raw reads matrix to find out differentially expressed genes. Genes with absolute fold change not less than 2, and adjusted P-value less than 0.05 were defined as differentially expressed genes.

\section{Statistical analysis}

Statistical significance was determined using Student's t test or Wilcox test and was assumed at $\mathrm{P} \square<\square 0.05$. Quantitative data are presented as the means $\square \pm \square$ se, as indicated in the figure legends. Statistical analyses were performed using R 4.10.

\section{Results}

\section{Identification of candidate variants for the consanguineous family with DCM}

A consanguineous family with three members affected DCM (family-1) was recruited. The proband from family-1 was diagnosed with DCM (NYHA class II). The results of conventional and dynamic electrocardiograms suggested occassionally first-degree atrioventricular block, paroxysmal sinus tachycardia and complete left bundle branch block (Fig. 1A). Increased circulating brain natriuretic peptide(BNP) and mildly elevated troponin were also observed. A sibling of the proband was diagnosed with severe DCM, when ze presented with a short history of symptoms of congestive heart failure. Ze experienced a rapid deterioration and eventually died one year after surgery treatment. A sibling of the proband, IV-3, was diagnosed with DCM (NYHA class II ) after presentation to clinic with respiratory distress. Ze had a left ventricle ejection fraction (LV EF ) value of 55\%, slightly dilated LV(a diameter of $52 \mathrm{~mm}$ in 
left ventricular). All three DCM patients were children of consanguineous parents, but none of the parents or other ancestors showed cardiac dysfunction.

Whole-exome sequencing were performed on the consanguineous family members with DCM, but found no known DCM-causing gene mutations, we then turned to seek new candidate pathogenic genes. The analysis of high-throughput second-generation sequencing data proceeded as illustrated (Supplementary Fig. S1). In brief, we firstly filtered out nonharmful variations and acquired harmful variations for further analysis. Subsequently, common variants in East Asians, which defined as variations with population frequency $\geq 5 \%$ in the normal human population database consisting of The Thousand Genomes Project, ESP6500SI-V2, ExAC Human Exome Integration Database and in-house database, were further removed, leaving 14,208 variants for further selection. Considering autosomal recessive inheritance pattern of the consanguineous family, both DCM patients including the proband should be a homozygous affected individuals, and parents of the proband could be heterozygous carriers. And only fifty-six variants accorded with the autosome recessive genetic pattern were regarded as candidate disease-causing variants. Finally, fifty-one more variants were left out based on the American College of Medical Genetics and Genomics (ACMG) 2015 guidelines, resulting in five variants located at five separate genes, BICD2, SERINC1, BVES, ADCY1 and PAPPA for further evaluations (Table S1).

$B I C D 2$ is a dynein activating adaptor protein involved in microtubule-based minus-end-directed transport via docking the dynein motor proteins to appropriate cargos. Lower LMNA protein expression and $M K L-1 / S R F$ activity were observed in Bicd2-deficiency mice reported previously by Dick et al[20]. It is known that LMNA is the most common causative gene for DCM, accounting for $4 \%-8 \%$ of patients with DCM[26-28]. In addition, some individuals with spinal muscular atrophy caused by the BICD2 'hot spot' mutation, c.302C>T:p.Ser107Leu, surprisingly presented the 
heart failure associated symptom, exertional and supine dyspnoea[18]. Considering the possible interaction relationship between $L M N A$ and BICD2, and the heart failure symptom that BICD2 mutant patient exhibited, we hypothesized that the homozygous variant (exon7:c.G2429:p.R810H) in C-terminal region of BICD2 (Table S1) would be a candidate variant that caused DCM in this family.

The missense variant of BICD2 (exon7: c.2429G>A:p.Arg810His) (Table S1), segregated with the disease phenotype. Prediction score of the SIFT(sorting intolerant from tolerant) algorithm[29] was 0.003(Supplementary Fig. S2), and the variant was considered to be damaging to protein function. Sanger sequencing was further utilized to validate the variant (exon7: c.2429G>A:p.Arg810His) in DCM patients from family 1 . Sanger results confirmed that both parents were heterozygous carriers and the two surviving DCM patients (the proband and sibling) were homozygous at this locus (Fig. 1B). Furthermore, the amino acid affected by this variant are highly evolutionarily conserved in not only H. sapiens but also Macaca mulatta, Bos taurus, rattus norvegicus, Mus musculus, Gallus gallus, and Danio rerio (Fig. 1C). It is well known that evolutionarily conserved sites in a multiple sequence alignment usually correspond to functionally important sites. Therefore, the missense variant in the evolutionarily conserved site (encoding amino acid R) could be harmful to protein function.

\section{Verification of three BICD2 variants from 210 sporadic DCM cases}

Patients hospitalized at the Cardiology Department of Sichuan People's Hospital at least once for heart failure from 2014 to 2017 were enrolled in our functional research about BICD2. We further selected patients diagnosed with DCM and patients diagnosed with abnormal loading conditions, coronary artery disease, hypertension, valvular disease or congenital heart disease. Following the above criteria, we obtained in total 210 sporadic DCM patients for further BICD2 mutant validation. 
In 210 sporadic DCM cases, we performed full exon sequencing of BICD2 and found one missense variant and two synonymous variants (Fig.2). The missense variant located in exon 2 (c.421G>A, p.Arg141Ser) (Fig. 2A) contributed to amino acid shifting from arginine to serine. Arginine is a basic amino acid and serine is an uncharged amino acid. Besides, algorithm prediction of functional impact suggested that it was a deleterious variant (Table S2). But no information about this variant could be acquire in normal population databases including The Thousand Genomes Project, ESP6500SI-V2, ExAC Human Exome Integration Database, gnomeAD, and ESP Exome sequencing project. The other two variants of BICD2 were Synonymous (Table S2). These results support that BICD2 may paly a significant role in DCM pathogenesis.

\section{Expression status of $B I C D 2$ in heart tissue}

We explored to quantify expression of $B I C D 2$ in heart of human as well as mouse and zebrafish. Real-time PCR results showed that Bicd2 mRNA expressed in hearts of 1-week, 4-week old C57 mice, with increasing expression level upon age (Fig. 3A). Similar expression pattern was also observed in hearts of zebrafish and expression of bicd 2 measured by qPCR was much higher at stage of 7 months than that at stage of 5 months (Fig. 3B).

Subsequently, we further detected the expression of BICD2 at the protein level. Immunohistochemical staining of BICD2 in normal heart tissue (left ear) supported its high expression in human(Fig. 3C). Moreover, BICD2 bulk RNA-seq data from GTEx database confirmed its relatively higher expression in human heart (Fig. 3D), and scRNA-seq data of BICD2 suggested that it mainly expressed in endothelial cells, fibroblasts, immune cells (Supplementary Fig. S3). Furthermore, published scRNA-seq (GSE95140) of cardiomyocytes from human hearts revealed that BICD2 expressed slightly higher in DCM and that in control (median RPKM of BICD2 in DCM and control is 2.33 and 2.28)(Fig. 3E), implicating a vital role of BICD2 in 
regulating DCM cardiomyocytes. Thus, we demonstrated that BICD2 expressed in hearts of human, and mouse and zebrafish at both mRNA and protein levels.

We also conducted in situ hybridization of bicd 2 in zebrafish at all three embryonic stages to explore its expression status (Fig. 3F). At 14hpf, bicd2 expressed in the axial midline cells of embryos. In detail, weak expression were observed in the somatic segment and strong expression were witnessed in the caudal notochord. At 24hpf, expression of bicd 2 was observed in eye, ventral part of the 4th ventricle, and hindbrain of zebrafish. At $48 \mathrm{hpf}$, bicd 2 expressed in the retina, forebrain, midbrain and hindbrain regions, with strong expression in the pectoral fin bud base (Fig. 3F). Lateral and abdominal views of embryos showed that bicd 2 was weakly expressed in the heart region. Taken together, BICD2/Bicd2/bicd2 expressed at heart of multiple species, implying its involvement in heart development and function.

\section{Knockout of bicd2 lead to partial embryonic lethality in homozygotes and altered cardiac function}

To further investigate the molecular mechanism of BICD2 in the pathogenesis of DCM, we designed a bicd2 knockdown assay in zebrafish to unravel associated phenotypic changes, and the experimental workflow is shown in Fig. 4A. We injected Cas9/sgRNA into embryos of F0 generation zebrafish, and further screened for bicd2-deficiency zebrafish in F0 adult zebrafish (Fig. 4A)(CRISPR target sequence in Table S3). Three fish were obtained, and they were mated with each other to further screened bicd 2 heterozygous F1 generations(Fig. 4A)(PCR primers for F1 mutant identification in Table S4). Details for generation of bicd2-KO zebrafish is depicted in Supplementary Fig. S4. In detail, we identified one type of mutation for F1 zebrafish, with 42-bp deletion (42bp deletion and 2bp insertion) at target sites, causing gene frame shift and induce early termination of the encoded protein. We then allowed the F1 generation fish to self-cross to get F2 generation fishes with three segregating genotypes (Fig. 4A). We observed the F2 generation fish in 2 different periods, i.e. 
embryonic stage and adult stage. In the embryonic period, we mainly observed the survival rate of embryos of different genotypes, and we measured the survival rate of embryos in three periods: 50hdf, 76hdf and 120hdf (Fig. 4A). Considering the insufficient number of homozygotes, we observed three batches of zebrafish at three different embryonic stages. At the adult stage, we performed echocardiographic measurements and transcriptome sequencing to compare bicd2-deficient to wild-type fish to delineate possible regulatory mechanisms underlying the cardiac dysfunction caused by bicd2 (Fig. 4A).

We firstly examined bicd2 by immunostaining to check whether bicd2 efficiently knocked out from zebrafish hearts. And immunostaining images of the bicd2-deficiency fish showed lower bicd2 expression than the wild-type group (Fig. 4B). We then analyzed number of viable embryos and found that the number of viable embryos was much lower in homozygotes than that in the other two groups, at all three time points (Fig. 4C, Supplementary Fig. S5). At 50hpf, there was only one homozygous embryo survived (Supplementary Fig. S5) (Table S5), while the number for heterozygotes and wild-type fish were twenty-two and thirteen(Supplementary Fig. S5) (Table S5), respectively. The proportion of viable homozygous embryos was $2.78 \%$, far less than the theoretical ratio $25 \%$. Similar trend was observed at both 76hpf and $120 \mathrm{hpf}$, and the proportion of viable homozygous embryos was $15 \%$ and 6.25\%(Fig. 4C, Supplementary Fig. S5) (Table S5), respectively. The embryonic viability data we observed for different genotypes suggests that bicd2 plays a vital role in zebrafish embryogenesis and supports the conclusion that bicd2 partially lead to death. We also examined heart rate of these three genotype groups at 50hdf, $76 \mathrm{hdf}$ and 120hdf, but we found no significant difference (Supplementary Fig. S6) (Table S6). Besides, no obvious phenotype was observed during embryo development period (images not shown). Then we turned to analyze the corresponding metrics of cardiac function at adult stage.

Electrocardiography was performed on wild-type and homozygous adult fish at seven 
months. In zebrafish echocardiographic studies, ventricular chamber size has mainly been assessed using volume and area. We therefore measured two-dimensional end-diastolic area (VAd) and end-systolic area (VAs), and three-dimensional end-diastolic volume (EDV), and end-systolic volume (ESV) in both wild-type and homozygous zebrafish. Compared with wild-type zebrafish, the bicd2-- fish, showed slightly larger value in both VAd and VAs (Fig. 4D, Table S7). EF was much lower in the bicd2-deficiency group than that in wild-type group (Wilcox test, P-value $=0.063$ ) (Fig. 4D, Table S7). It is well known that EF is a measurement of a percentage of how much blood the left ventricle pumps out with each contraction, and calculated as the ratio of amount of blood pumped out to amount of blood in chamber. $\mathrm{EF}<45 \%$ is a diagnosis measurement for DCM[3]. And in our studies, most homozygotes presented $\mathrm{EF}<45 \%$, supporting that bicd 2 was crucial for cardiac function. What's more, cardiac output was significantly lower in the bicd2-- fish than that in wild-type fish (Wilcox test, P-value <0.05) (Fig 7A, Table S7). In addition, the bicd2-- fish showed lower stroke volume (Fig. 4D, Table S7). Echocardiographic images represented zebrafish hearts with two different genotypes were extracted from echocardiogram and were comparably listed (Fig. 4E). In summary, these results indicate that bicd2 knockout may cause abnormal contraction of the heart.

\section{RNA-seq of bicd2-deficient zebrafish revealed cardiomyocyte}

\section{transcriptome shift}

We conducted transcriptome sequencing in hearts of bicd 2 knockout zebrafish and wild-type zebrafish to examine the shifts in their transcriptional profiles. Consistent with expectations, bicd2 expression was significantly lower in the knockout group than in the wild-type group (Table S8). Gene Set Enrichment Analysis (GSEA)[30] based on ranking of all expressed genes could discern more telltale biological clues than enrichment analysis solely focused on differential expressed genes (DEGs). Therefore, we firstly utilized GSEA to unravel significantly enriched gene sets. 
medRxiv preprint doi: https://doi.org/10.1101/2022.02.03.22269864; this version posted February 4, 2022. The copyright holder for this preprint (which was not certified by peer review) is the author/funder, who has granted medRxiv a license to display the preprint in perpetuity.

All rights reserved. No reuse allowed without permission.

Compared to the wild-type group, the bicd2-homozygous group showed enrichment for cardiopathy-related signaling pathways and metabolic pathways, for example KEGG_CARDIAC_MUSCLE_CONTRACTION, KEGG_CALCIUM_SIGNALING _PATHWAY, KEGG_OXIDATIVE_PHOSPHORYLATION, KEGG_GLYCOLYSIS GLUCONEOGENESIS, KEGG_CITRATE_CYCLE_TCA_CYCLE and KEGG_PEN

-TOSE_PHOSPHATE_PATHWAY(Fig 5A), as well as pathways associated with nervous system disease (Table S9). The results accord with previous functional exploration of Bicd2 in mouse, which proved a causal role of Bicd2 in neuronal disorders, a dominant mild early onset form of spinal muscular atrophy[20]. Besides, pathways relate to energy metabolism in the mitochondria like oxidative phosphorylation (OXPH) and tricarboxylic acid cycle (TCA), are common characteristics of distinct heart failure demonstrated previously [31, 32]. Interestingly, many pathways (6 out of 14, 42.86\%) (Fig. 5B) increased in both bicd2 homozygous and heterozygous groups, including KEGG_CALCIUM_SIGNALING_PATHWAY and KEGG_GLYCOLYSIS_GLUCONEOGENESIS. Only 3 common pathways (Fig 5C) decreased in those two groups, implicating different transcriptome atlas between homozygous and heterozygous groups.

We then extract DEGs to further delineate biological meaningful pathways involved in bicd 2 gene regulatory network. Same as expected, the homozygotes displayed the largest change in all three groups, and in total 1708 DEGs (483 up-regulated genes , 1225 down-regulated genes, Fig. 5D, Table S10). Surprisingly, difference between the two bicd2-KO groups was much bigger than that between heterozygotes and the wild-type fish. In detail, there were 1645 DEGs (417 up-regulated genes , 1228 down-regulated genes, Fig 5D, Table S11) between homozygotes and heterozygotes, while 535 DEGs (334 up-regulated genes, 201 down-regulated genes, Fig 5D, Table S12) between heterozygous and wild-type groups. The top 15 enriched GO pathways based on 483 up-regulated and 1225 down-regulated genes in the homozygous group were displayed separately (Supplementary Fig. S7-8). Genes increased in the 
homozygous group mainly consisted of blood vessel development, extracellular matrix organization, cell-cell adhesion, carbohydrate metabolic process and circulatory system process (Supplementary Fig. S7). However, Genes decreased mainly related to the immune system, including regulation of immune system process, response to biotic stimulus, leukocyte activation (Supplementary Fig. S8).Notably, increased expression of genes encoding extracellular matrix proteins in DCM patients was previously demonstrated by microarray analysis[31]. In our study, extracellular matrix genes(Fig. 5E)(Supplementary Fig. S9) like col4a2, col4a4, col5a1, col8alb, coll $8 a 1 b$, and genes of circulatory system process (Fig. 12) showed higher expression in the homozygous group than that in wild-type. Genes related to regulation of immune system process (Fig. 11) like $c d 74 b$ and $c d 79 a$ decreased in the two bicd2-KO groups, implying activation of the immune system after knocking out bicd2 in zebrafish.

Fifty-one genes with human genetic evidence were proposed as causing susceptibility to inheritable DCM by previous studies[6]. Ortholog retrieval in the zfin data report (http://zfin.org/downloads/file/human_orthos.txt) and Ensembl (http://www.ensembl.o -rg/index.html) identified zebrafish homologues for 48 of these genes(Table S13). Among these 48 genes, 28 had a single orthologue in zebrafish (Supplementary Fig. S 12, Table S13). 8 out of 28 orthologs including bag3, nebl, psen2, jph2, dtna, psen1, gatadl and plekhm2 showed the lowest expression in the homozygous group(Supplementary Fig. S12). On the contrary, 10 out of 28 orthologs including mybpc3, lama4, nppa, tnni3k, cmlc1, nexn, lrrc10, csrp3, abcc9, tcap showed the highest expression in the homozygous group(Supplementary Fig. S12). There were also 20 human DCM genes that harbored multiple orthologues in zebrafish (Supplementary Fig. S13).

\section{Discussion}

In this study, whole-exome sequencing of a consanguineous family with 3 DCM 
patients revealed a missense mutation (exon7: c.2429G>A: p.Arg810His) in BICD2 was the corresponding pathological variant. Consanguineous couples of the family were both heterozygous carriers, and two DCM patients were homozygous genotype at this locus. Harmful effect of this mutation on amino acids were supported by algorithms, and the amino acid affected by these three variants are highly evolutionarily conserved in multiple species including Mus musculus, and Danio rerio, facilitating the functional exploration of the variant in mouse and zebrafish. Besides, genetic screening of BICD2 in 210 sporadic DCM cases revealed three more variants of $B I C D 2$, further demonstrating the significance of $B I C D 2$ involved in DCM.

Most previously reported familiar DCM cases are caused by pathogenic variants inherited in autosome dominant pattern[5]. Many DCM-linked genes encode proteins of the sarcomere, costamere, $\mathrm{Z}$ band, and nuclear membrane[5]. Currently more than 250 genes spanning >10 gene ontologies have been suggested contributing to inherited DCM[6]. However, heritability of DCM could not all be explained by variants discovered so far. In a consanguineous family we collected in clinical, the pathological variant for DCM was searched and a novel DCM-linked gene BICD2 was discovered. Notably, the inheritance mode of DCM in the consanguineous family was autosome recressive pattern, suggesting loss of function caused the illness.

$B I C D 2$ is a dynein activating adaptor protein that plays a critical role in microtubule-based minus-end-directed transport[13, 14]. Bicd2-KO mice showed significantly lower $L M N A$ expression at protein level, decreased $M K L-1 / S R F$ activity, and significantly downregulated expression of its downstream $\alpha$-actin, $\alpha$-coactin and membrane association proteins, and microtubulin[20], thus affecting cardiovascular system development.

Furthermore, we conducted functional experiments in mouse and zebrafish to verify the relationship of BICD2 and DCM. Real-time PCR results confirmed that Bicd2 mRNA expressed in hearts of C57 mice and zebrafish. Immunostaining result showed 
that $B I C D 2$ expressed in hearts of clinical patients at the protein level. Further bicd2 knockdown assay in zebrafish revealed DCM-linked phenotypic changes. The survival rate of embryos in three periods, $50 \mathrm{hdf}, 76 \mathrm{hdf}$ and $120 \mathrm{hdf}$ revealed that bicd 2 is embryonic lethal, which is consistent with observations in Bicd2-KO in mice [20]. Different Ejection Fraction(EF) values between bicd2-deficiency and wild-type fishes was detected by echocardiography. RNA-seq of bicd2-KO revealed DEGs enriched in pathways relate to energy metabolism in the mitochondria like oxidative phosphorylation (OXPH) and tricarboxylic acid cycle (TCA), which are common characteristics of distinct heart failure demonstrated previously.

In this study, we identified a nonsynonymous pathological variant in BICD2 from a consanguineous family, and we further found other BICD2 variants associated with DCM, implying the significance role of BICD2 played in DCM. We then demonstrated that deletion of bicd2 in zebrafish decreased LVEF and altered energy metabolism in the mitochondria, implicating cardiac function dysfunction. Our study shows that $B I C D 2$, an adapter protein linking the dynein motor complex to various cargos, is a novel DCM candidate gene and conceptually expands our horizons regarding pathogenesis of DCM. To the best of our knowledge, this is one of the few reports of consanguineous family in the cardiovascular disease DCM, as evidenced by WES data, echocardiography, and zebrafish models.

However, we temporarily do not prove the direct relationship between the exact BICD2 variant (exon7:c.G2429:p.R810H) and phenotypes associated to enlarged heart. We will continue to explore the cardiac dysfunction led by the BICD2 variant. In addition, we will continue to validate the presumably pathologic variant in $B I C D 2$ on more DCM patients in the future to provide additional evidence.

\section{Conclusions}

Our study shows that $B I C D 2$, an adapter protein linking the dynein motor complex to various cargos, is a novel DCM candidate gene and conceptually expands our 
horizons regarding pathogenesis of DCM.

\section{Abbreviations}

DCM, dilated cardiomyopathy; WES, whole-exome sequencing; BICD2, Bicaudal-D2; $\mathrm{LV}$, left ventricular; RNA-seq, RNA-sequencing; EF, ejection fraction; FS, fractional shortening; PAC, frequent premature atrial contractions; NYHA, the New York Heart Association; ACMG, the American College of Medical Genetics and Genomics; DEG, differentially expressed genes

\section{Authors' contributions}

Kai Luo: Conception and design, Collection and assembly of data, Data analysis and interpretation, Manuscript writing; Chenqing Zheng, Rong Luo: Provision of study material, Data analysis and interpretation; Xin Cao, Huajun Sun: Provision of study material, Assembly of data; Huihui Ma, Jichang Huang, Yang Xu: Responsible for manuscript writing and revision of the manuscript; Xiushan $\mathrm{Wu}$ : Collection and assembly of data, Data analysis and interpretation, Final approval of manuscript; Xiaoping Li: Conception and design, Manuscript writing, Financial support, Final approval of manuscript. Xiaoping Li is the guarantor of this work and, as such, had full access to all the data in the study and takes responsibility for the integrity of the data and the accuracy of the data analysis. All authors read and approved the final manuscript.

\section{Funding}

This work was supported by grants from Chinese National Natural Science Foundation (No. 81770379, 32171182, 81470521, and 81670290), and the Foundation of Chengdu Medical College (CYZZD21-04, 2021LHPJ-02).

\section{Declaration of competing interest}

None

\section{Data Availability}

The datasets used during the study are available are available within the article and its Supplementary materials.

\section{Disclosures}




\section{Acknowledgements}

The authors would like to thank all patients who provided samples used in this study.

\section{Reference}

1. Douglas P. Zipes MD PLMP, Robert O. Bonow MD MS, Douglas L. Mann MD, Gordon F. Tomaselli MD: Braunwald's Heart Disease: A Textbook of Cardiovascular Medicine, vol. 11th Edition; 2018.

2. Towbin JA, Lowe AM, Colan SD, Sleeper LA, Orav EJ, Clunie S, Messere J, Cox GF, Lurie PR, Hsu D et al. Incidence, causes, and outcomes of dilated cardiomyopathy in children. JAMA 2006; 296(15):1867-1876.

3. McNally EM, Mestroni L. Dilated Cardiomyopathy: Genetic Determinants and Mechanisms. Circ Res 2017; 121(7):731-748.

4. Maron BJ, Towbin JA, Thiene G, Antzelevitch C, Corrado D, Arnett D, Moss AJ, Seidman CE, Young JB, American Heart A et al. Contemporary definitions and classification of the cardiomyopathies: an American Heart Association Scientific Statement from the Council on Clinical Cardiology, Heart Failure and Transplantation Committee; Quality of Care and Outcomes Research and Functional Genomics and Translational Biology Interdisciplinary Working Groups; and Council on Epidemiology and Prevention. Circulation 2006; 113(14):1807-1816.

5. McNally EM, Golbus JR, Puckelwartz MJ. Genetic mutations and mechanisms in dilated cardiomyopathy. J Clin Invest 2013; 123(1):19-26.

6. Jordan E, Peterson L, Ai T, Asatryan B, Bronicki L, Brown E, Celeghin R, Edwards M, Fan J, Ingles $\mathrm{J}$ et al. Evidence-Based Assessment of Genes in Dilated Cardiomyopathy. Circulation 2021; 144(1):7-19.

7. Harakalova M, Kummeling G, Sammani A, Linschoten M, Baas AF, van der Smagt J, Doevendans PA, van Tintelen JP, Dooijes D, Mokry M et al. A systematic analysis of genetic dilated cardiomyopathy reveals numerous ubiquitously expressed and muscle-specific genes. Eur J Heart Fail 2015; 17(5):484-493.

8. Hershberger RE, Morales A, Siegfried JD. Clinical and genetic issues in dilated cardiomyopathy: a review for genetics professionals. Genet Med 2010; 12(11):655-667.

9. Burkett EL, Hershberger RE. Clinical and genetic issues in familial dilated cardiomyopathy. $J$ Am Coll Cardiol 2005; 45(7):969-981.

10. Lefeber DJ, de Brouwer AP, Morava E, Riemersma M, Schuurs-Hoeijmakers JH, Absmanner B, Verrijp K, van den Akker WM, Huijben K, Steenbergen G et al. Autosomal recessive dilated cardiomyopathy due to DOLK mutations results from abnormal dystroglycan O-mannosylation. PLoS Genet 2011; 7(12):e1002427.

11. Venugopalan P, Agarwal AK, de Bono D. Low proportion of familial dilated cardiomyopathy 
in an arab population with a high prevalence of consanguineous marriages. Acta Paediatr 2001; 90(11):1267-1270.

12. Levitas A, Muhammad E, Zhang Y, Perea Gil I, Serrano R, Diaz N, Arafat M, Gavidia AA, Kapiloff MS, Mercola M et al. A Novel Recessive Mutation in SPEG Causes Early Onset Dilated Cardiomyopathy. PLoS Genet 2020; 16(9):e1009000.

13. Hoogenraad CC, Wulf P, Schiefermeier N, Stepanova T, Galjart N, Small JV, Grosveld F, de Zeeuw CI, Akhmanova A. Bicaudal D induces selective dynein-mediated microtubule minus end-directed transport. EMBO J 2003; 22(22):6004-6015.

14. Hoogenraad CC, Akhmanova A, Howell SA, Dortland BR, De Zeeuw CI, Willemsen R, Visser P, Grosveld F, Galjart N. Mammalian Golgi-associated Bicaudal-D2 functions in the dynein-dynactin pathway by interacting with these complexes. EMBO $J$ 2001; 20(15):4041-4054.

15. Peeters K, Litvinenko I, Asselbergh B, Almeida-Souza L, Chamova T, Geuens T, Ydens E, Zimon M, Irobi J, De Vriendt E et al. Molecular defects in the motor adaptor BICD2 cause proximal spinal muscular atrophy with autosomal-dominant inheritance. Am J Hum Genet 2013; 92(6):955-964.

16. Oates EC, Rossor AM, Hafezparast M, Gonzalez M, Speziani F, MacArthur DG, Lek M, Cottenie E, Scoto M, Foley AR et al. Mutations in BICD2 cause dominant congenital spinal muscular atrophy and hereditary spastic paraplegia. Am J Hum Genet 2013; 92(6):965-973.

17. Neveling K, Martinez-Carrera LA, Holker I, Heister A, Verrips A, Hosseini-Barkooie SM, Gilissen C, Vermeer S, Pennings M, Meijer R et al. Mutations in BICD2, which encodes a golgin and important motor adaptor, cause congenital autosomal-dominant spinal muscular atrophy. Am J Hum Genet 2013; 92(6):946-954.

18. Rossor AM, Oates EC, Salter HK, Liu Y, Murphy SM, Schule R, Gonzalez MA, Scoto M, Phadke R, Sewry CA et al. Phenotypic and molecular insights into spinal muscular atrophy due to mutations in BICD2. Brain 2015; 138(Pt 2):293-310.

19. McKenney RJ, Huynh W, Tanenbaum ME, Bhabha G, Vale RD. Activation of cytoplasmic dynein motility by dynactin-cargo adapter complexes. Science 2014; 345(6194):337-341.

20. Jaarsma D, van den Berg R, Wulf PS, van Erp S, Keijzer N, Schlager MA, de Graaff E, De Zeeuw CI, Pasterkamp RJ, Akhmanova A et al. A role for Bicaudal-D2 in radial cerebellar granule cell migration. Nat Commun 2014; 5:3411.

21. Ho CY, Jaalouk DE, Vartiainen MK, Lammerding J. Lamin A/C and emerin regulate MKL1-SRF activity by modulating actin dynamics. Nature 2013; 497(7450):507-511.

22. Parlakian A, Charvet C, Escoubet B, Mericskay M, Molkentin JD, Gary-Bobo G, De Windt LJ, Ludosky MA, Paulin D, Daegelen D et al. Temporally controlled onset of dilated cardiomyopathy through disruption of the SRF gene in adult heart. Circulation 2005; 112(19):2930-2939.

23. Ding Y, Sun X, Huang W, Hoage T, Redfield M, Kushwaha S, Sivasubbu S, Lin X, Ekker S, $\mathrm{Xu} \mathrm{X}$. Haploinsufficiency of target of rapamycin attenuates cardiomyopathies in adult zebrafish. Circ Res 2011; 109(6):658-669.

24. Pertea M, Kim D, Pertea GM, Leek JT, Salzberg SL. Transcript-level expression analysis of RNA-seq experiments with HISAT, StringTie and Ballgown. Nat Protoc 2016; 11(9):1650-1667.

25. Leng N, Dawson JA, Thomson JA, Ruotti V, Rissman AI, Smits BM, Haag JD, Gould MN, 
Stewart RM, Kendziorski C. EBSeq: an empirical Bayes hierarchical model for inference in RNA-seq experiments. Bioinformatics 2013; 29(8):1035-1043.

26. Fatkin D, MacRae C, Sasaki T, Wolff MR, Porcu M, Frenneaux M, Atherton J, Vidaillet HJ, Jr., Spudich S, De Girolami U et al. Missense mutations in the rod domain of the lamin A/C gene as causes of dilated cardiomyopathy and conduction-system disease. N Engl J Med 1999; 341(23):1715-1724.

27. Parks SB, Kushner JD, Nauman D, Burgess D, Ludwigsen S, Peterson A, Li D, Jakobs P, Litt M, Porter CB et al. Lamin A/C mutation analysis in a cohort of 324 unrelated patients with idiopathic or familial dilated cardiomyopathy. Am Heart J 2008; 156(1):161-169.

28. Pugh TJ, Kelly MA, Gowrisankar S, Hynes E, Seidman MA, Baxter SM, Bowser M, Harrison B, Aaron D, Mahanta LM et al. The landscape of genetic variation in dilated cardiomyopathy as surveyed by clinical DNA sequencing. Genet Med 2014; 16(8):601-608.

29. Vaser R, Adusumalli S, Leng SN, Sikic M, Ng PC. SIFT missense predictions for genomes. Nat Protoc 2016; 11(1):1-9.

30. Subramanian A, Tamayo P, Mootha VK, Mukherjee S, Ebert BL, Gillette MA, Paulovich A, Pomeroy SL, Golub TR, Lander ES et al. Gene set enrichment analysis: a knowledge-based approach for interpreting genome-wide expression profiles. Proc Natl Acad Sci U S A 2005; 102(43):15545-15550.

31. Haywood ME, Cocciolo A, Porter KF, Dobrinskikh E, Slavov D, Graw SL, Reece TB, Ambardekar AV, Bristow MR, Mestroni L et al. Transcriptome signature of ventricular arrhythmia in dilated cardiomyopathy reveals increased fibrosis and activated TP53. J Mol Cell Cardiol 2020; 139:124-134.

32. Sweet ME, Cocciolo A, Slavov D, Jones KL, Sweet JR, Graw SL, Reece TB, Ambardekar AV, Bristow MR, Mestroni L et al. Transcriptome analysis of human heart failure reveals dysregulated cell adhesion in dilated cardiomyopathy and activated immune pathways in ischemic heart failure. BMC Genomics 2018; 19(1):812.

\section{Fig. legends}

\section{Fig. 1. Family pedigree and clinical phenotypes.}

A. Echocardiography and electrocardiogram images of the proband showing dilated heart and decreased LVEF. Upper left panel: parasternal long-axis echocardiogram view of the left ventricle showing two-dimensional measurements of right and left ventricular wall thickness, septal thickness and right and left ventricular internal diameter. Upper right panel: apical four chamber echocardiogram view showing length, area, volume, end-systolic volume(ESV) and ejection fraction of the left ventricle. Lower panel: 12 lead electrocardiogram(ECG) showing paroxysmal sinus tachycardia and complete left bundle branch block.

B. Sanger sequencing results indicating the genotypes of the family members. 
Arrows indicate the variant locus.

C. Protein sequence alignment of amino sequences surrounding the BICD2 variant with orthologues from $\mathrm{H}$. sapiens to Danio Rerio. Note that the amino acid sequences surrounding the affected amino acid residues are highly conserved.

Fig. 2. Validation of BICD2 in 210 sporadic DCM patients.

A. Three BICD2 variants identified in 210 sporadic DCM patients.

B. Sanger sequencing results of the missense variant.

Fig. 3. Expression of BICD2 in hearts of human, mouse and zebrafish.

A. qPCR results of Bicd2 expression in hearts of C57 mouse suggesting an increasing trend over developmental stages $(n=3)$. Data are represented as the mean $\pm S D$.

B. qPCR results of BICD2 expression in hearts of zebrafish suggesting an increasing trend over developmental stages $(n=3)$. Data are represented as the mean $\pm S D$.

C. Immunohistochemistry with anti-BICD2 antibody of normal human heart showing ubiquitous staining.

D. Bulk RNA-seq revealed mRNA expression (transcript per million fragments) of BICD2 in multiple tissues from human.

E. BICD2 expression difference in scRNA-seq of cardiomyocytes from $\operatorname{DCM}(n=213)$ and control( $\mathrm{n}=52)$ deposited in GSE95140. Student $\mathrm{t}$ test, pvalue=0.063.

F. In situ hybridization of bicd 2 in zebrafish revealed that bicd2 expressed in hearts at embryonic stage.

\section{Fig. 4. DCM associated phenotypes in bicd2-deficient zebrafish}

A. Flow chart of bicd2 zebrafish models for functional experiment.

B. Immunohistochemistry with anti-BICD2 antibody of zebrafish hearts showing the absence of staining in bicd2-- fishes and ubiquitous staining in wild-type fishes.

C. Number of embryos in homozygotes $(n=2)$, heterozygotes $(n=16)$ and wild-type 
fishes(n=14) at embryonic stage of $120 \mathrm{hdf}$.

D. Area, volume, Ejection Fraction, Fractional shortening, cardiac output, and stroke volume difference in homozygotes $(n=5)$, and wild-type fishes $(n=4)$.

E. Echocardiogram plot showing ejection fraction difference in a homozygote and a wild-type fish.

Fig. 5. bicd2-deficient hearts exhibited cardiac transcriptome shift

A. GSEA plots of six KEGG pathways showing higher expression in bicd2-zebrafish.

B. Venn diagrams showing enriched KEGG pathways increased in the bicd2-deficiency groups.

C. Venn diagrams showing enriched KEGG pathways decreased in the bicd2-deficiency groups.

D. Differential expressed genes in the two bicd2-deficiency groups. Cells are filled according to z-score: red indicates higher (activated), blue indicates lower (inhibited).

E. Differential expressed genes in extracellular matrix organization. Cells are filled according to z-score: red indicates higher (activated), blue indicates lower (inhibited).

F. Differential expressed genes in circulatory system process. Cells are filled according to z-score: red indicates higher (activated), blue indicates lower (inhibited).

G. Differential expressed genes in regulation of immune system process. Cells are filled according to z-score: red indicates higher (activated), blue indicates lower (inhibited). 

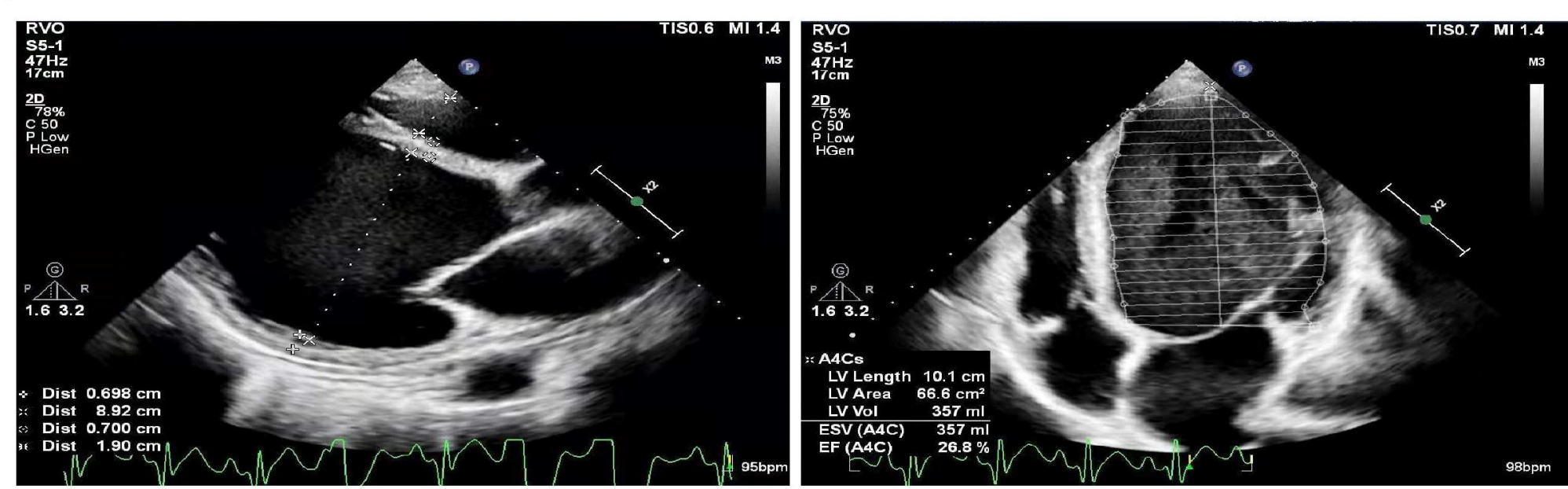

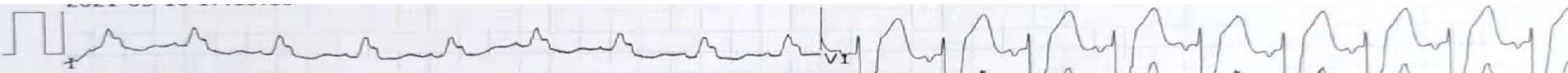
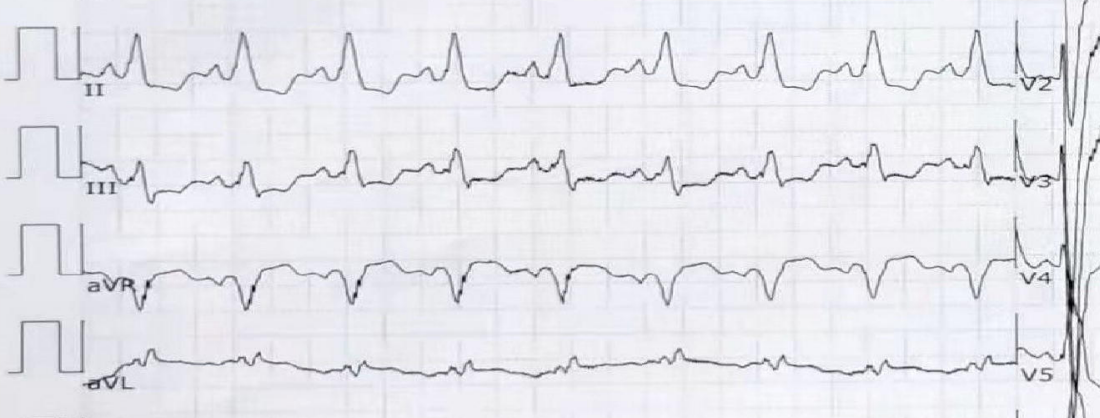

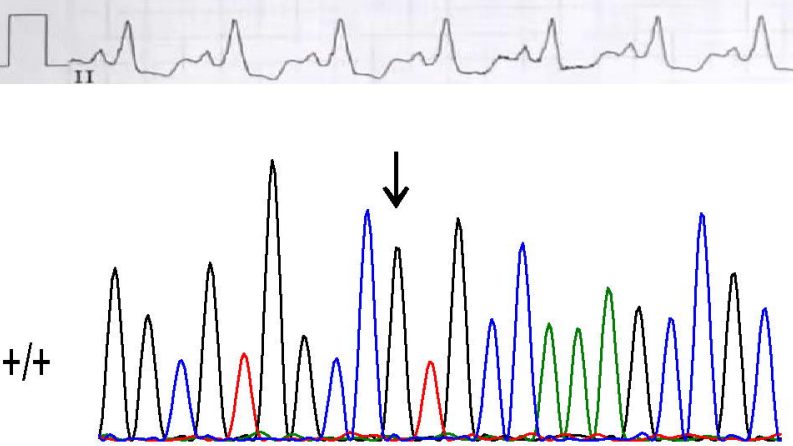

G G C G T G G C C G T G C C A A A G C C G C

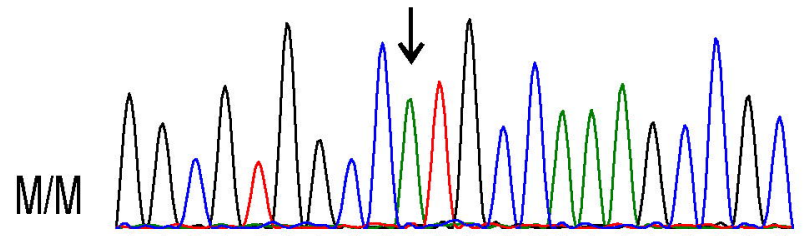

G G C G T G G C CA T G C C A A A G C C G C

C

$\sqrt{-}$ Homo sapiens HEQTRBGRAKAAP

L Maca camulatta HEQTRBGBT/ AAP/A

Danio rerio VIKEGWGERTER SAEE

- Gallus gallus HEQSKAVTKSAS/AK

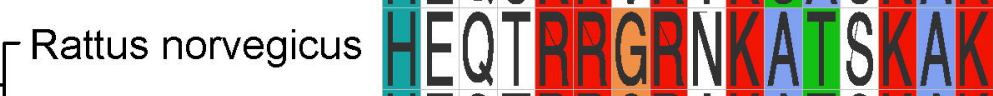

Bos taurus

Mus musculus

HEQTRR G A ATS AK

HEQTRGG SIAASEAK 


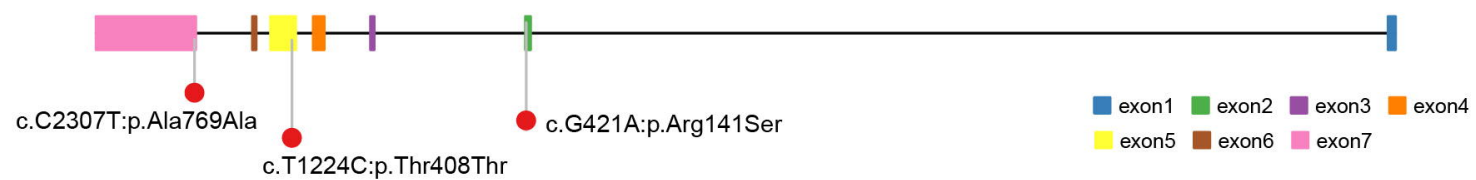

B

c.G421A:pR141S

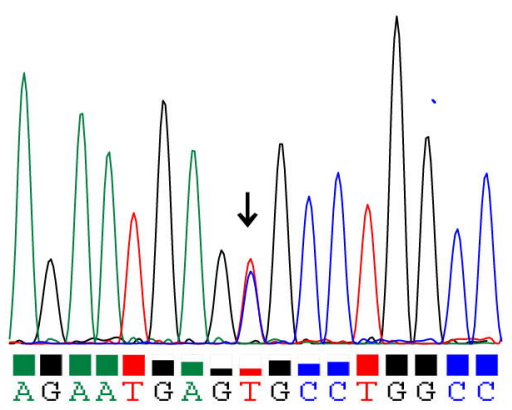

c.T1224C:pT141T

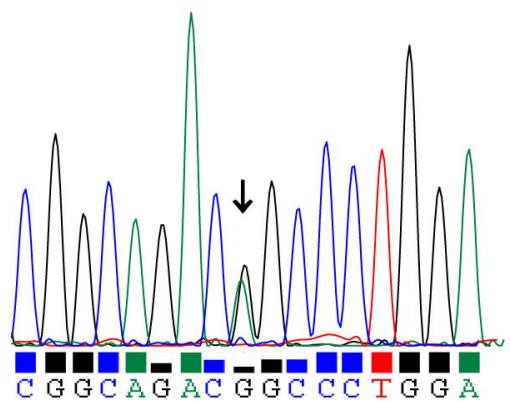

c.C2307T:pA141A

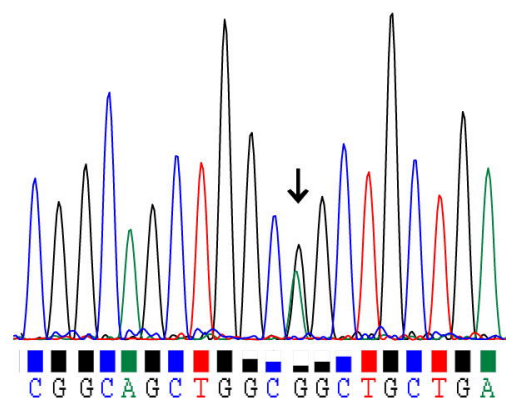



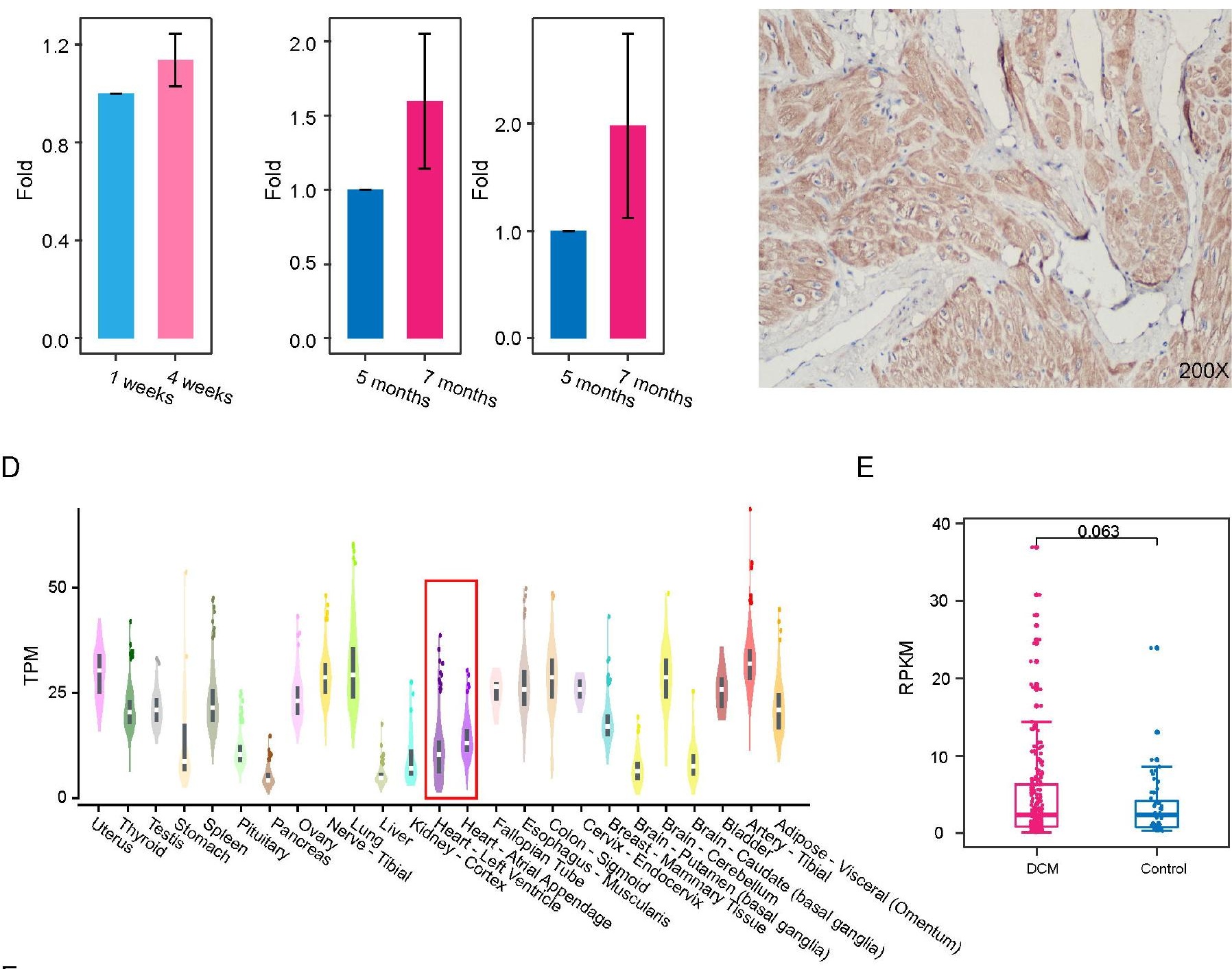

E

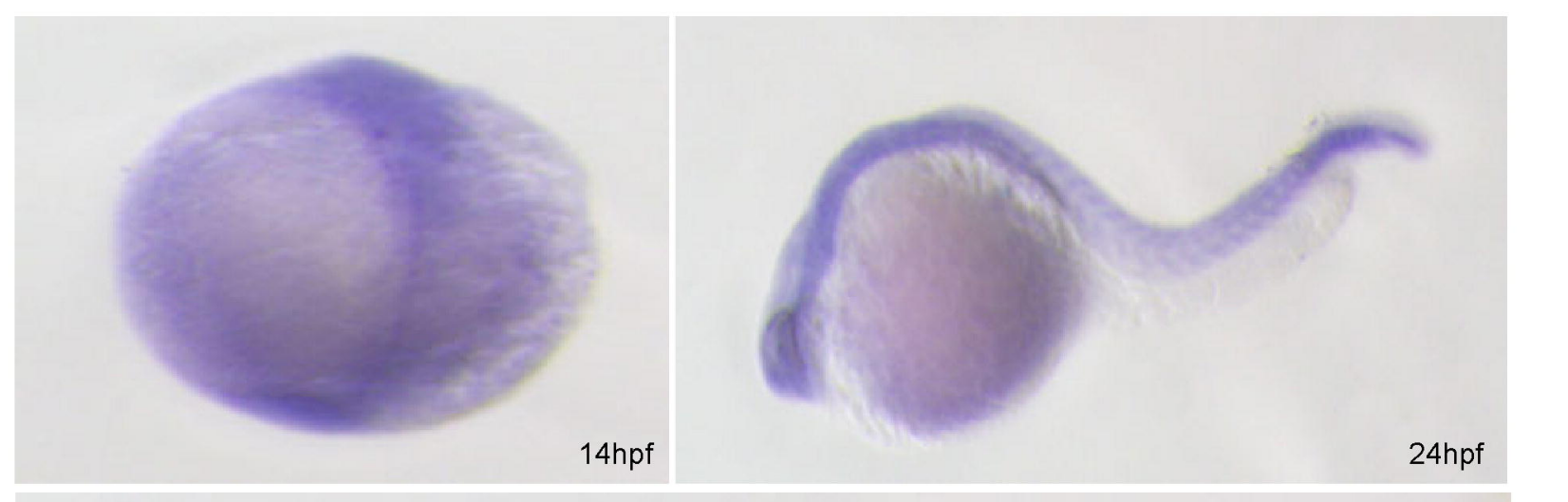


A

Fo embryo

Cas9/sgRNA injection

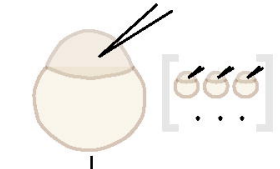

F0 adult zebrafish

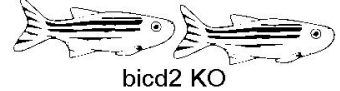

bicd2 KO

F1 adult zebrafish

$\otimes$

bicd2 KO (genotype:+-)

F2

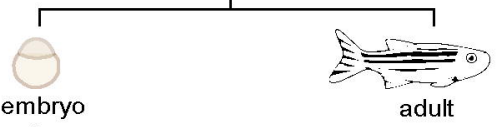

++ vs +- vs --

50hpf 76hpf 120hpf

RNA-seq echocardiogram

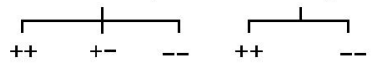

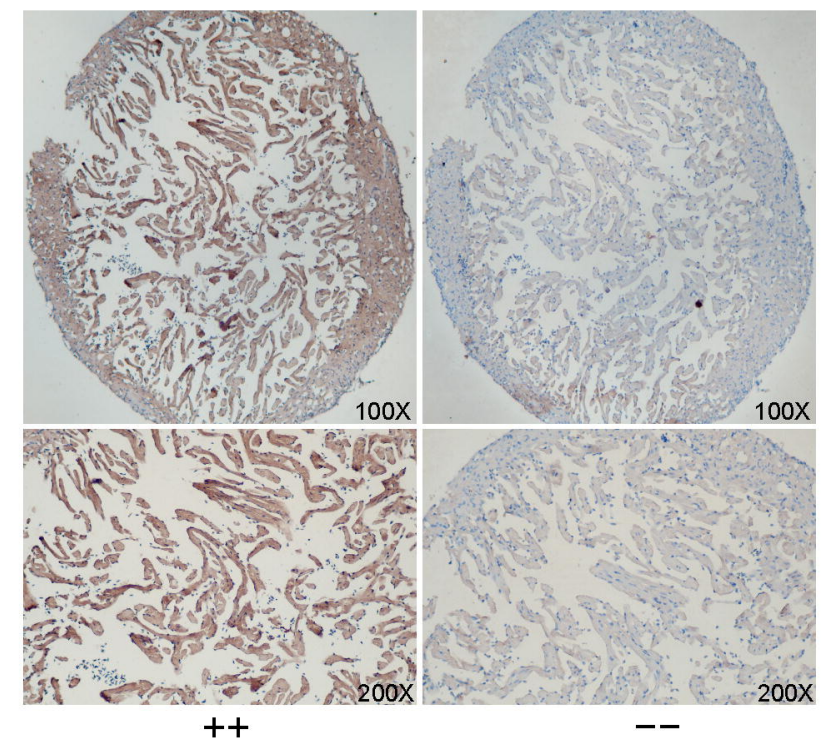

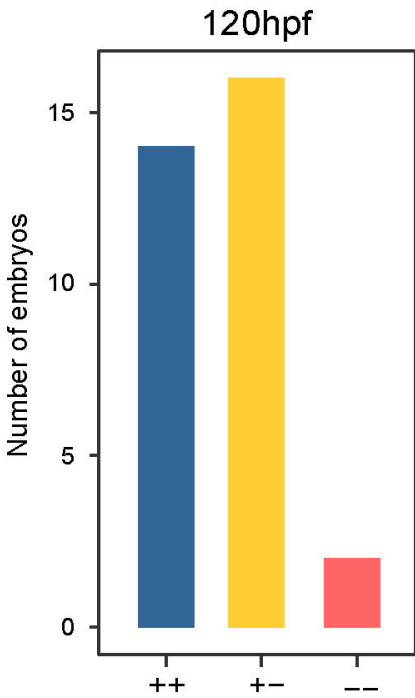

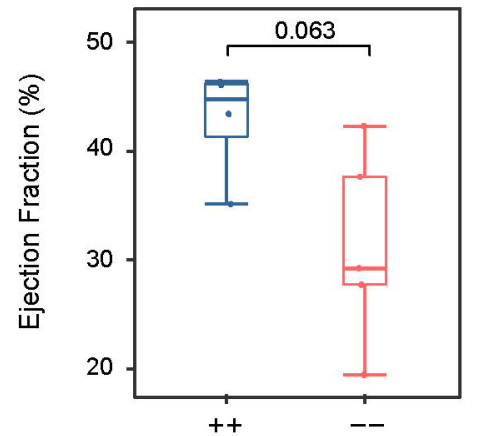
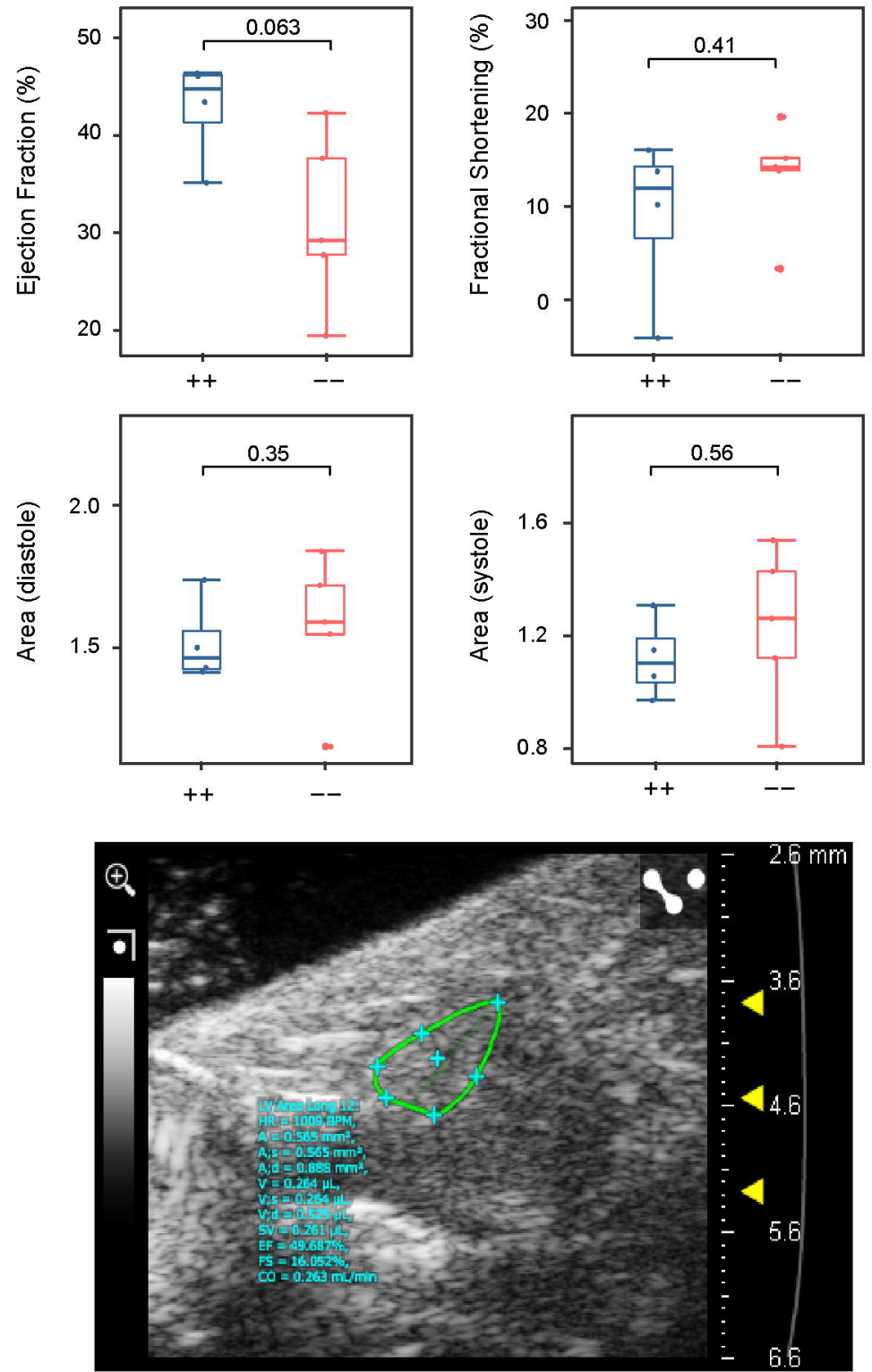

$++$
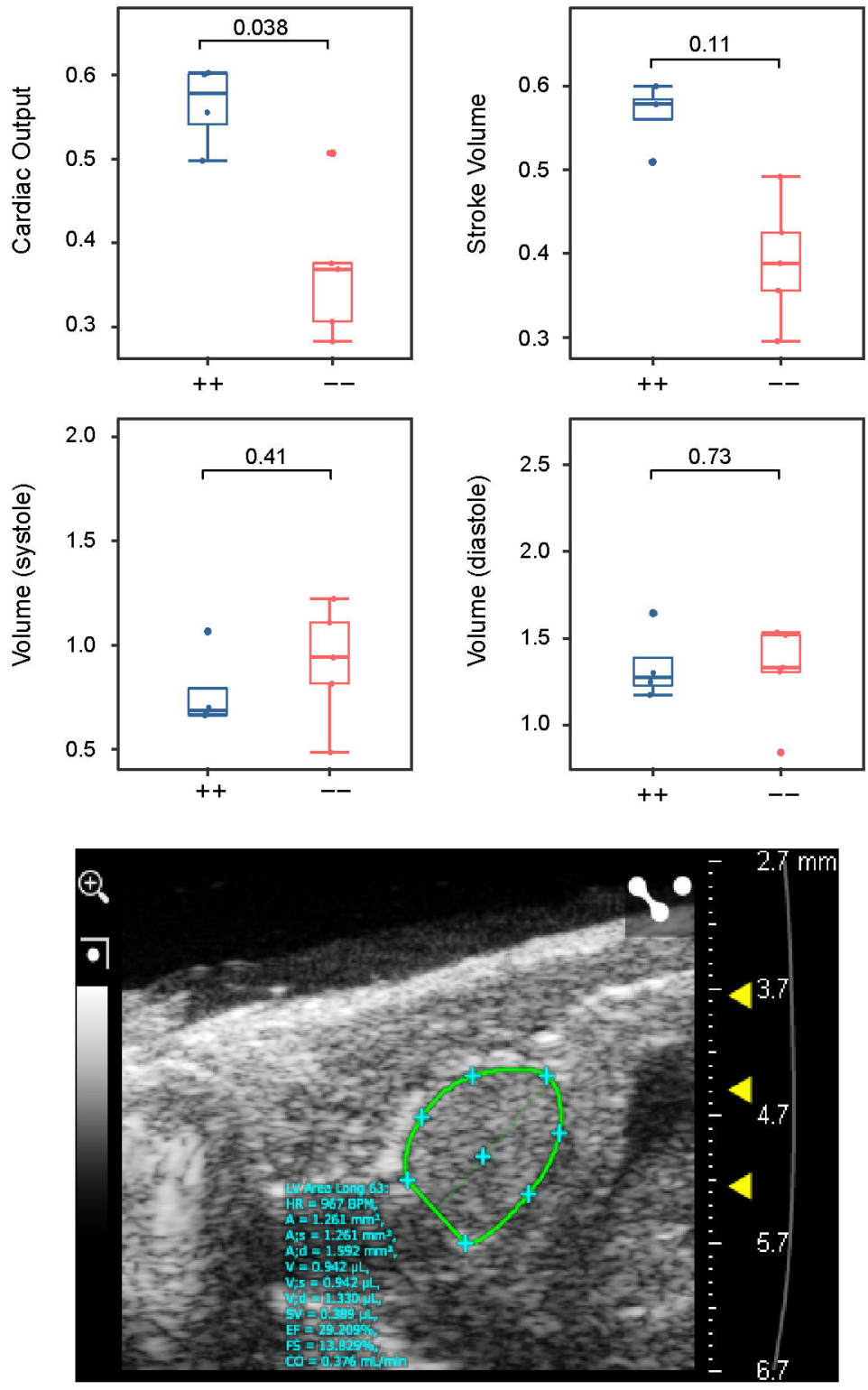

$--$ 
